\title{
Research on Prediction Methods of Wellbore Hydrate Formation of Ultra-Deep Gas Wells in Northwest Sichuan
}

\author{
Qilin Liu *, Jian Yang, Lang Du, Jianxun Jiang, Dan Ni, Rong Zheng, Zhaoqian Luo, Peng Liu \\ Northwest Sichuan Gas Mine of Petro China Southwest Oil \& Gasfield Company, Sichuan, China;
}

\begin{abstract}
According to the formation and handling situation of hydrate in ultra-deep high-pressure sulfurcontaining gas wells in northwest Sichuan, the formation conditions of natural gas hydrate was studied based on previous studies on hydrate, the molecular dynamics of natural gas hydrate and the multiphase flow law of high-temperature high-pressure high-sulfur-containing gas wellbore were combined, and the pressure prediction model with high-temperature high-pressure sulfur-containing gas wells as the target was built. The chemical and physical control methods of wellbore hydrate plugging were discussed to provide the scientific theoretical basis for the prediction and control of hydrate in high-temperature high-pressure high-sulfurcontaining gas wells.
\end{abstract}

Keywords: high-temperature and high-pressure, sulfur-containing gas well, wellbore, hydrate, prediction method.

\section{Introduction}

There are many oil and gas-containing reservoirs in the northwest Sichuan, at present, exploration and development targets are mainly the Qixia, Maokou, Changxing and Feixianguan group, gas wells show ultradeep, high-pressure and sulfur-containing characteristics, stratum pressure ranges from 91.13 to $131.09 \mathrm{MPa}$, stratum temperature ranges from 121.70 to $164.11^{\circ} \mathrm{C}$, hydrogen sulfide content range from 0.02 to $88 \mathrm{~g} / \mathrm{m} 3$, and total depth of four stratums all exceed $5500 \mathrm{~m}$, at present, the total depth in the Shuangyushi block is average $7746 \mathrm{~m}$, which is the record of the deepest wells of land average total depth of CNPC. The actual drilling confirmed that most of the new wells put into production in the above four stratums belong to ultra-deep high-pressure sulfurcontaining gas wells [1].

The hydrate formation temperature at the wellhead of ultra-deep high-pressure sulfur-containing gas wells in northern Sichuan is as high as about $35^{\circ} \mathrm{C}$, which is much higher than that of normal-pressure gas wells without hydrogen sulfide, and it seriously affects safe production. Recently, the wellbore hydrate blockage was formed during the production process, and after the measures of pumping glycol and wellhead insulation were ineffective, finally, the self-heating unblocking process was adopted to remove the blockage, the operation from start to finish is a total of 5 months, affecting the output $1812 \times 10^{4} \mathrm{~m}^{3}$; it is easy to form hydrate and freeze plugging in the production process; wellbore hydrate blockage is formed in the process of oil test, which affects the project progress; after opening the safety valve in the pit and preparing to pump ethylene glycol, hydrate blockage was rapidly formed in the wellbore (within 2 hours), affecting the normal production.

There are still gaps between the current theoretical research and field practice in hydrate formation mechanism and law, the degree of influence of pressure, hydrogen sulfide content, gas-liquid ratio, wellbore working conditions and other factors on hydrate formation and accurate prediction of hydrate formation process and time, which need further research, and provide theoretical basis and guiding basis for the injection system of injecting hydrate inhibitor in the wellbore [2]. In wellbore hydrate control, in addition to the existing self-heating unblocking process that needs further optimization; exploration and research on other ultra-deep high-pressure sulfur-containing gas wellbore unblocking processes need to be carried out.

\section{Present Situation of Hydrate Prediction at Home and Abroad}

The long-term research has been carried out at home and abroad in the prediction of hydrate formation conditions, for effective hydrate control schemes, several thermodynamic prediction models of hydrate formation conditions have been proposed and applied in natural gas development at home and abroad, including van der Waals-Platteeuw model, improved version of vdW-P model (Parrish-Prausnitz, Ng- Robinson model), and Chen-Guo model, etc. On the basis of CSMHyK hydrate

\footnotetext{
* Corresponding author: liuq110@petrochina.com.cn
} 
kinetic model and OLGA multiphase flow equation, JingYi Shi and Jian-Chun Fan et al. built the numerical simulation method of hydrate formation in deep water drilling, and predicted the formation scope and degree of wellbore annulus hydrate by introducing the degree of supercooling density to characterize the hydrate formation possibility [3]. Antonin Chapoy et al. Heriot-Watt University, UK, achieved good results in describing the phase equilibrium of natural gas hydrates by modifying the vdW-P model. Peter Jorgensen Herslund et al. Technical University of Denmark built the model that can describe the phase equilibrium of natural gas hydrates containing $\mathrm{CO}_{2}$ based on the CPA equation of state and the vdW-P model, and he obtained high computational accuracy by adsorbing this model with modified binary interaction parameters and Langmuir. Ioannis Tsivintzelis, Technical University of Denmark, M. Naveed Khan, Colorado School of Mines, and other scholars have studied the association effect containing $\mathrm{H}_{2} \mathrm{~S}$ and other polar components, which provides the references for the modification of the association parameters of $\mathrm{H}_{2} \mathrm{O}, \mathrm{H}_{2} \mathrm{~S}$, $\mathrm{CO}_{2}$, and alcohol materials. Therefore, on the basis of CPA equation of state and vdW-P model, predicting the formation conditions of sour gas hydrates has a good application in sour gas fields, modifying binary interaction parameters or association parameters in polar molecules such as $\mathrm{H}_{2} \mathrm{~S}$, this is expected to solve the difficult problem of predicting gas hydrate formation conditions in ultra-deep high-pressure sulfur-containing gas wells in northwest Sichuan [4].

\section{Formation Conditions of Natural Gas Hydrate}

The formation of hydrate is not only related to the component, composition and free water content of natural gas, but also needs certain thermodynamic conditions, namely a certain temperature and pressure. Russian scholar Rozeboom et al. believed that only when the pressure of gas components in the system is greater than its hydrate decomposition pressure, the gas containing saturated water vapor can spontaneously generate hydrate[5], which can be expressed by fugacity as follows:

$$
f_{\text {decomposition }}^{\text {hydrate }}<f_{M}^{\text {system }} \leq f_{M}^{\text {saturation }}
$$

The relative density of natural gas composed of different components is different, natural gas with relatively high density is more likely to form hydrate [6]. The main conditions of forming hydrate are: partial free water in the natural gas fluid and fluid temperature is less than or equal to the dew point of free water. The fluid is prone to generate hydrate when it passes through the pipeline, throttling components or ground equipment with intense disturbance.

\section{Prediction of High-Temperature High- Pressure Wellbore Hydrate}

\subsection{Research on flow law of sour gas in wellbore}

According to the formation conditions of hydrate, when predicting where hydrates are likely to form and how much they will crystallize during production, it is necessary to calculate the temperature and pressure of the fluid at every moment in the wellbore; therefore, the study of wellbore multiphase flow law of sulfur-containing gas wells is the prerequisite for predicting hydrate formation. The flow pressure and temperature change of fluid in the wellbore vary greatly, the parameter calculation methods of Hagedorn brown and other multiphase flow correlation formulas do not consider the change of wellbore phase state, amd the parameters are obtained based on the method of crude oil, in order to make Hagedorn Brown model and other correlation formulas more suitable for multiphase flow calculation of gas wells containing water and $\mathrm{H}_{2} \mathrm{~S}$, the parameters in the correlation formula should consider the characteristics of wellbore phase change[7], and the correction of phase change should be considered under the influence of temperature, pressure and other factors. This problem is still explained based on the Hagedorn Brown correlation formula.

$$
\frac{\Delta p}{\Delta h}=g \rho_{m} \sin \theta+\frac{f_{m} q_{L}{ }^{2} M_{t}{ }^{2}}{9.21 \times 10^{9} d^{5} \rho_{m}}
$$

According to previous studies, there are two dissolution mechanisms of sulfur in natural gas with high $\mathrm{H}_{2} \mathrm{~S}$ content, namely physical dissolution and chemical dissolution. In the following, relevant theories of association models in supercritical fluids are first used to explain these two dissolution mechanisms, and then the association model containing two dissolution actions is built [8]-[10].

$$
\frac{y}{(1-y)^{n}}=\left[\frac{k \varphi_{1}^{n} \varphi_{s}^{s} P_{s}^{s} \exp \left(\int_{P_{s}^{s}}^{P} \frac{V_{s}^{s}}{R T} d p\right)}{\varphi_{3}}\right] P^{n-1}
$$

In the formula:

$V_{s}^{s}$------------Molar volume of solid solute sulfur;

$P_{s}^{s}$------------Saturated vapor pressure of solid solute sulfur;

$\varphi_{s}^{s}$------------Fugacity coefficient of pure solid solute sulfur;

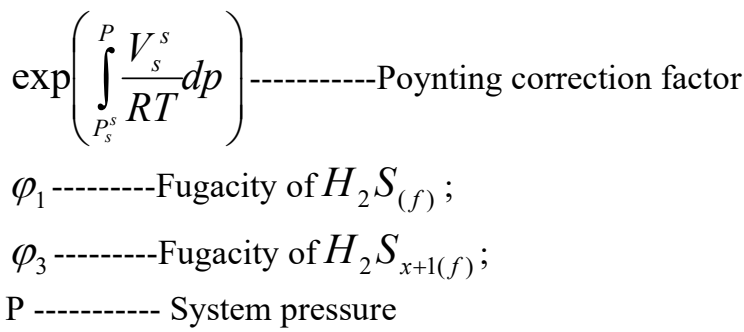


The fugacity of solid is calculated by the following formula:

$$
f_{s}^{s}=\varphi_{s}^{s} P_{s}^{s} \exp \left(\int_{P_{s}^{s}}^{P} \frac{V_{s}^{s}}{R T} d p\right)
$$

\subsection{Prediction research on hydrate formation}

\subsubsection{Diagram method}

In mine practice, the commonly used empirical diagram method is based on hydrate formation temperature and pressure curve calculated in accordance with relative density of natural gas, combine the calculation results of natural gas with different relative densities, thus, the relationship curve between temperature and pressure of forming gas hydrate is drawn. The empirical diagram method can be used to predict the approximate formation area of hydrate. The empirical diagram method can be used as the judgment basis and plays a key role in rough prediction of the formation area of gas hydrate[11]. The following figure can be used as the basis for judging hydrate formation.

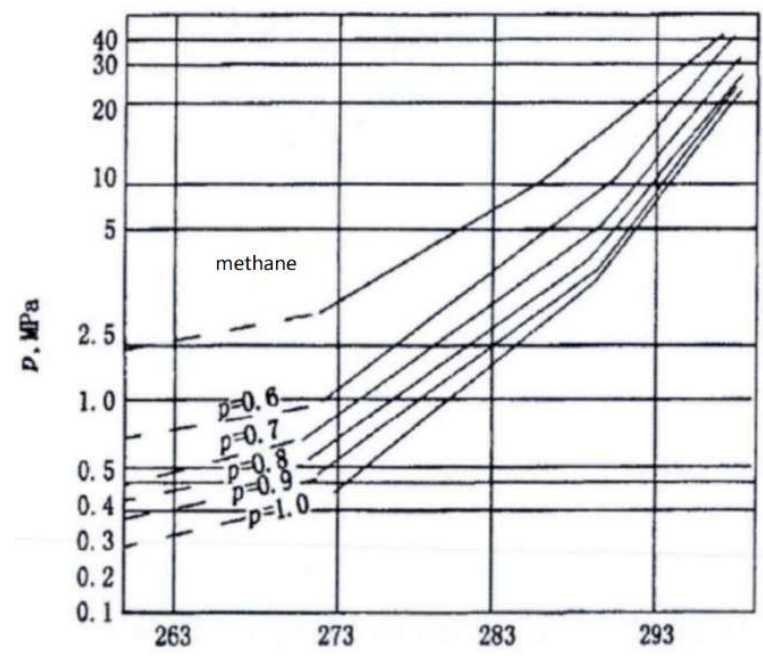

Fig.1 Predicted P-T phase diagram of relative density and hydrate formation

The figure gives the temperature and pressure curves of methane and five natural gas fluids with 0.6, 0.7, 0.8, 0.9 and 1.0 relative density, respectively. If the relative density of natural gas is known to fall in the curves in the figure, the formation conditions of hydrate can be determined by linear interpolation.

Given $\mathrm{R}$ and $\mathrm{T}$, when $\mathrm{P}$ is generated by hydrate, the formula is:

$$
P=P_{1}+\left[\left(P_{1}-P_{2}\right) \frac{r_{1}-r}{r_{1}-r_{2}}\right]
$$

Given $\mathrm{R}$ and $\mathrm{P}$, when $\mathrm{T}$ is generated by hydrate, the formula is:

$$
T=T_{1}+\left[\left(T_{2}-T_{1}\right) \frac{r_{1}-r}{r_{1}-r_{2}}\right]
$$

In the formula, $r--$ the relative density of natural gas, $r_{1}<r<r_{2}$
$P_{1}, P_{2}$ formation pressure of gas hydrate with relative density $r_{1}, r_{2}$;

$T_{1}, T_{2}$ - formation temperature of gas hydrate with relative density $r_{1}, r_{2}$.

\subsubsection{Statistical thermodynamics method}

The prediction algorithm of hydrate formation is derived by statistical thermodynamics method via Newton's method[12], The general form of the equation is:

$$
\ln \mathrm{Z}-\mathrm{Y}=0
$$

For natural gas without $\mathrm{H}_{2} \mathrm{~S}$, when $\mathrm{P}>6.865 \mathrm{MPa}$ :

$$
\lg Z=8.9751-0.03304 \mathrm{~T}
$$

When $\mathrm{P}<6.865 \mathrm{MPa}$ when:

$$
\lg \mathrm{Z}=3.51517-0.01436 \mathrm{~T}
$$

For natural gas with $\mathrm{H}_{2} \mathrm{~S}$ :

For Y:

$$
\lg Z=-5.40964-0.02133 \mathrm{~T}
$$

$$
\mathrm{Y}=\gamma_{1} \ln \left(1-\sum_{1}^{n} \theta_{A 1}\right)+\gamma_{2} \ln \left(1-\sum_{1}^{n} \theta_{A 2}\right)
$$

$\theta_{\mathrm{A} 1}$ and $\theta_{\mathrm{A} 2}$ reflect the filling degree of gas in the large and small cavity of hydrate, respectively, which can be expressed as:

$$
\theta_{i j}=\frac{C_{i j} P_{i}}{1+C_{1 j} P_{j}+C_{2 j} P_{j}}
$$

In the formula, $\mathrm{Cij}$ can be calculated as follows:

$$
C_{i j}=\exp \left(A_{i j}-B_{i j} T\right)
$$

In the formula, the values of $\mathrm{A}_{\mathrm{ij}}$ and $\mathrm{B}_{\mathrm{ij}}$ coefficients are shown in Table 1.

Table.1 Relationship between $\mathrm{A}_{\mathrm{ij}}$ and $\mathrm{B}_{\mathrm{ij}}$ and natural gas components

\begin{tabular}{|c|c|c|c|c|}
\hline \multirow{2}{*}{$\begin{array}{c}\text { natural gas } \\
\text { component } \mathrm{j}\end{array}$} & \multicolumn{2}{|c|}{ small hole } & \multicolumn{2}{c|}{ big hole } \\
\cline { 2 - 5 } & $\mathrm{A}_{1 \mathrm{j}}$ & $\mathrm{B}_{1 j}$ & $\mathrm{~A}_{2 j}$ & $\mathrm{~B}_{2 j}$ \\
\hline $\mathrm{CH}_{4}$ & 6.0499 & 0.02844 & 6.2957 & 0.02845 \\
\hline $\mathrm{C}_{2} \mathrm{H}_{6}$ & 9.4892 & 0.04058 & 11.9410 & 0.04180 \\
\hline $\mathrm{C}_{3} \mathrm{H}_{8}$ & -43.670 & 0.00000 & 18.2760 & 0.04661 \\
\hline $\mathrm{C}_{4} \mathrm{H}_{10}$ & -43.670 & 0.00000 & 13.6942 & 0.02773 \\
\hline $\mathrm{N}_{2}$ & 3.2485 & 0.0262 & 7.5990 & 0.02447 \\
\hline $\mathrm{CO}_{2}$ & 23.0350 & 0.0904 & 25.2710 & 0.09781 \\
\hline $\mathrm{H}_{2} \mathrm{~S}$ & 4.9285 & 0.0093 & 2.4030 & 0.00633 \\
\hline
\end{tabular}

The hydrate formation temperature under a given pressure is calculated and solved by Newton's method, the iterative format is:

$$
T^{n+1}=T^{n}-\frac{f\left(T^{n}\right)}{f^{\prime}\left(T^{n}\right)}
$$

\subsubsection{Van der Waals-Platteeuw model}

In 1959, van der Waals and Platteeuw et al. [13] proposed the thermodynamic model based on the phase equilibrium theory. That is:

$$
\mu_{\mathrm{w}}^{\mathrm{H}}=\mu_{\mathrm{w}}^{\alpha}
$$

In the Formula: $\mu_{\mathrm{w}}^{\mathrm{H}}$-- chemical potential of phase $\mathrm{H}$;

$\mu_{\mathrm{w}}^{\alpha}$-- chemical potential in the water-rich phase.

The chemical potential of hydrate lattice ( $\beta$ phase) as the reference state, the equilibrium condition can be expressed as:

$$
\Delta \mu_{\mathrm{w}}^{\mathrm{H}-\beta}=\Delta \mu_{\mathrm{w}}^{\alpha-\beta}
$$


The thermodynamic model of water-rich phase is as follows:

(1) Hydrate phase

Van der Waals and Platteeuw derived the expression of chemical potential difference $\Delta \mu^{\mathrm{H}}$ based on statistical thermodynamics and isothermal adsorption theory:

$$
\Delta \mu_{\mathrm{W}}^{\mathrm{H}}=\mu_{\mathrm{W}}^{\mathrm{H}}-\mu_{\mathrm{W}}^{\beta}=-R T \sum_{m=1}^{2} \gamma_{m} \ln \left(1-\sum_{j} Y_{j m}\right)
$$

In the formula: -- chemical potential of the fully empty hydrate, $\mathrm{J} / \mathrm{mol}$;

$\mu^{H}$-- Chemical potential of fully filled hydrate, $\mathrm{J} /$ mol;

$\gamma_{m^{--}}$Characteristic constant of hydrate structure, for structure I: $\gamma_{1}$ take $1 / 23, \gamma_{2}$ take $3 / 23$; For structure II: $\gamma_{1}$ take $2 / 17, \gamma_{2}$ take $1 / 17$

$R$-- Gas constant, $8.31434 \mathrm{~J} /(\mathrm{mol} \bullet \mathrm{K})$;

$T$-- temperature, $\mathrm{K}$;

$Y_{j m}$-- The fraction of $m$ type hole occupied by $j$ group.

The fraction $Y_{j m}$ of $m$ type hole occupied by $j$ group is calculated based on the following formula:

$$
Y_{j m}=\frac{C_{j m} f_{j}}{1+\sum_{j} C_{j m} f_{j}}
$$

In the formula: $f_{j}$-- Fugacity of $j$ component, MPa;

$C_{j m}$-- Langmuir constant of the $j$ component in the $m$ type hole, $1 / \mathrm{MPa}$.

In the above formula, the expression of Langmuir constant $C_{\mathrm{jm}}$ was calculated by using the empirical relation proposed by Parrish and Prausnitz[14], thus simplifying the cumbersome steps of vDW-P model in solving $C_{\mathrm{jm}}$, The expression of $C_{\mathrm{jm}}$ is shown as follows:

$$
C_{j m}=\frac{A_{j m}}{T} \exp \left(\frac{B_{j m}}{T}\right)
$$

In the formula, $A_{j m}, B_{j m}$ - experimental fitting parameters. Most of the fitting parameters of structure I and II hydrates in this model come from studies by Munck[15], Rasmussen and Pedersen et al. [25].

(2) Water-rich phase

Holder et al. believed that the chemical potential deviation between the fully empty hydrate lattice in the water-rich phase and the water phase was only related to temperature, and proposed the following expression $\Delta \mu_{\mathrm{W}}^{\beta-\alpha}$

$$
\frac{\Delta \mu_{\mathrm{W}}^{\mathrm{\beta} \cdot \mu}}{R T}=\frac{\Delta \mu_{\mathrm{W}}^{0}}{R T_{0}}-\int_{T_{0}}^{T}\left(\Delta h_{\mathrm{W}}^{0}+\int_{T_{0}}^{T} \Delta C_{P \mathrm{~W}}^{0} \mathrm{~d} T\right) \frac{\Delta h_{\mathrm{W}}}{R T^{2}} \mathrm{~d} T+\int_{0}^{P} \frac{\left(\Delta V_{\mathrm{W}}^{0}+\Delta V_{\mathrm{W}}^{1} P\right)}{R T} \mathrm{~d} P-\ln x_{\mathrm{W}}
$$

In the formula: $\Delta h_{\mathrm{W}}^{0}$ and $\Delta C_{\mathrm{PW}}^{0}$--represent the enthalpy difference $(\mathrm{J} / \mathrm{mol})$ and heat capacity difference $[\mathrm{J} /(\mathrm{mol} \cdot \mathrm{K})]$ of phase $\beta$ and phase $\alpha$ at $\mathrm{T}_{0}\left(\mathrm{~T}_{0}=273.15 \mathrm{~K}\right)$, respectively;

$$
\Delta V_{\mathrm{W}}^{0} \text { and } \Delta V_{\mathrm{W}}^{\mathrm{d}--} \text { represent the molar volume }
$$
difference $\left(\mathrm{m}^{3} / \mathrm{mol}\right)$ under the reference condition $\left(T_{0}=273.15 \mathrm{~K}\right)$ and the actual condition, respectively, $\mathrm{m}^{3} / \mathrm{mol}$;

$x_{\mathrm{w}}$-- represents the molar fraction of water in the solution [13].

\section{Control Hydrate Blockage in Wellbore}

In the control of hydrate blockage in wellbore, the common ways are chemical unblocking and physical unblocking. Chemical unblocking is in hydrate inhibitors and unblocking solution research, methanol and ethylene glycol are commonly used at home in the early stage, but because the methanol has toxicity, and unblocking effect of ethylene glycol is not good, therefore, the inhibitors in the follow-up study mainly consider the needs of environmental protection and safety based on having the function of inhibiting hydrate, a new non-toxic hydrate inhibitor Z-6 has been developed and applied in Dongsheng Gas field, the field application is carried out, the results show that this new inhibitor can replace methanol in hydrate prevention and unblocking effect[16].

The coiled tubing operation is usually used in physical unblocking, however, The coiled tubing operation of ultra-deep high-pressure sulfur-containing gas wells has high requirements for coiled tubing strength, sulfur resistance, injection head capacity, coiled tubing performance, control of jacking force at dredging moment, well control device, operation tools in the pit, performance of sand washing fluid, friction pump pressure and risk identification and control during operation [17]. In the early stage of Tarim Oilfield, in allusion to the problems such as deep buried reservoir (6500 8038m), high temperature $\left(150 \sim 190{ }^{\circ} \mathrm{C}\right)$, high stratum pressure (105 136mpa) and difficult coiled tubing operation in Kucheshan structure, the pressurized operation method was successfully used to solve the wellbore wax blockage, but the cost was high [18]. In order to solve the difficulties of coiled tubing operation, the coiled tubing unblocking process and supporting equipment suitable for Kucheshan had been successfully applied in Tarim Oilfield through software simulation calculation and process argument, and they were improved based on the liquid and process problems in the operation process, had been successfully applied to the follow-up oil tube blocking in high-temperature, highpressure and high sulfur-containing wells and achieved good results. 


\section{Conclusions and Suggestions}

The formation mechanism of gas hydrate and the research on wellbore flow law of high-temperature, high-pressure and high sulfur-containing gas wells were combined, the prediction method of wellbore hydrate formation in hightemperature, high-pressure and high sulfur-containing gas wells was built.

Three prediction methods of hydrate formation, diagram method, statistical potential method and phase equilibrium calculation method, were discussed, it is concluded that diagram method is convenient to determine the hydrate formation condition in the case of knowing temperature and pressure.

Statistical thermodynamics method and Van der Waals-Plateaus model are conducive and calculate the temperature and pressure of the whole wellbore section in real time to predict the formation of natural gas hydrate.

The usual approaches are chemical unblocking and physical unblocking in the control of wellbore hydrate blockage. The new non-toxic inhibitor Z-6 is suggested for chemical unblocking.

\section{Acknowledgments}

Scientific Project of Petro China Southwest Oil \& Gasfield Company "Research on Wellbore Risk Evaluation and Control Technology of Ultra-Deep and High-Pressure Sulfur-Containing Gas Wells in Northwest Sichuan", No. 20200303-24.

\section{References}

1. Deng Ke. Research on Hydrate Control Technology in Gas Field of West Sichuan[D]. Southwest Petroleum University, 2007.

2. Tan Linlin. Research on Effusion Law and Hydrate Control Technology of Surface Gathering and Transmission Pipeline in Dongsheng Gas Field[D]. Southwest Petroleum University, 2013.

3. Tang Daobing. Quantitative Research on Wellhead Temperature of Gas Wells to Prevent Hydrate Formation[J]. China Petroleum and Chemical Standard and Quality, 2018, 38 (07): 91-92 + 94.

4. Zhou Lianlian, Chen Lin, Lan Qiong, Xie Xulong, Wang Jia. Prevention and Application of Natural Gas Hydrate in Surface Gathering and Transportation of Longgang Gas Field[J] Natural Gas Technology and Economy, 2018, 12(01): 41-44+82-83.

5. $\mathrm{Xu}$ Changhui. Prediction and Control System Technology of Wellbore Hydrate in Gas Field Production System[D]. Yangtze University, 2016.

6. Liu Shixin. Experimental Study And Prediction of Gas Hydrate Control in Gas Field Production[D]. Southwest Petroleum Institute, 2005.

7. Huang Taihong, Wang Yang. Research on Prevention and Control Measures of Hydrate Ice Blockage in Gas Pipeline[J]. China Petroleum and
Chemical Standard and Quality, 2018, 38 (07): 141142.

8. Wang Li, Zhou Keming, et al. Experimental Investigation of Hydrate Forming Mechanism in Gas Reservoirs with High Sulfur Conten[J]. Natural Gas Industry, 2003.5, 23(3), 97-100.

9. Kennedy, H.T. and Wieland, D.R. Equilibrium in the Methane-Carbon Dioxide-Hydrogen Sulfide-Sulfur System, Traits, A1ME(1960\}21.

10. erdgas-zeitschrift, 99.jg., Heft 9, Septembet 1983, 297300.

11. Kunal Karan,Robert A.Heidemann and Leo A.Behie,Sulfur Solubility itt Sour Gas Predictions with an Equation of State Model,Ind.Eng.Chem.Res. 1998, 37, 1679-1684.

12. Yang Xuefeng, A Study On Special Phase Behavior State Of Multiphase Fluid In Gas Reservoirs With High Sulfur Content And Formation Damage Caused By The Sulfur Deposition[D]. Southwest Petroleum University.

13. Platteeuw J C, Waals J H V D. Thermodynamic properties of gas hydrates[J]. Molecular Physics, 1958, 1(1) :91-96

14. Parrish W R, Prausnitz J M. Dissociation Pressures of Gas Hydrates Formed by Gas Mixtures[J]. Ind. Eng.chem. process Design Devlop, 1972, 22(3) :2635.

15. Munck J, Skjold-J Rgensen S, Rasmussen P. Computations of the formation of gas hydrates[J]. Chemical Engineering Science, 1988, 43(10) :26612672.

16. Wu Zhiliang. Prediction Of Gas Hydrate Formation Conditions In Wellbore[J]. Petroleum Geology and Experiment, 2008(03):315-320.

17. Chen Yuchuan, Shi Bohui, Li Wenqing, Liu Yang, Song Shangfei, Ding Lin, Gong Jing. Progress Of Influence Mechanism Of Kinetic Hydrate Inhibitors[J]. Chemical Industry and Engineering Progress, 2018, 37(05): 1726-1743.

18. Miao Chunhui, Zhang Qinghu, Qiu Shujuan. Summarize the Formation of Gas Hydrates and its Prevention Technique in Long -Distance Transportation Pipeline[J]. Shanghai Gas, 2007(02): 23-25. 